\title{
Adaptation and Fatigue of a Mechanosensory Neuron in Wild-Type Drosophila and in Memory Mutants
}

\author{
Gabriel Corfas ${ }^{\mathrm{a}}$ and Yadin Dudai \\ Department of Neurobiology, The Weizmann Institute of Science, Rehovot 76100 , Israel
}

The mechanosensory neuron that innervates the anteronotopleural bristle of Drosophila melanogaster responds with a burst of action potentials to deflection of the bristle towards the body wall. The decay of the firing rate upon sustained deflection is typical of a slowly adapting mechanosensory neuron. Upon repeated monotonous stimulation, the response decreases and the kinetics of adaptation change; the response recovers after rest. This sensory fatigue depends on the duration of the stimuli and the rate of stimulation. Two mutants, rutabaga (rut) and dunce (dnc), which are defective in learning and in the activity of the CAMP cascade, show altered kinetics of sensory fatigue. In rut, which has a reduced CAMP synthesis, the mechanosensory neuron fatigues less rapidly, whereas in dnc, characterized by a reduced CAMP hydrolysis, the neuron fatigues more rapidly than in wild-type flies. The data suggest that the CAMP cascade plays a role in the mechanism of sensory fatigue. Our study shows, for the first time, the effect of memory mutations on functional properties of an identified neuron which subserves a modifiable behavior. The experimenta! system described here could also be useful for neurogenetic dissection of mechanosensory transduction.

Mechanosensory neurons innervating the macrochaetae that cover the notum of Drosophila melanogaster elicit a cleaning reflex upon deflection of the bristles (Vandervorst and Ghysen, 1980). Repetitive mechanical stimulation of the bristles results in reflex habituation (Corfas and Dudai, 1989a). In rutabaga (rut), a memory mutant lacking $\mathrm{Ca}^{2+} /$ calmodulin stimulation of adenylate cyclase (Dudai and Zvi, 1984; Livingstone et al., 1984), and in dunce ( $d n c)$, a memory mutant defective in cAMP phosphodiesterase (PDE) (Byers et al., 1981), memory of habituation of the cleaning reflex is abnormally short-lived (Corfas and Dudai, 1989a, b). The cleaning reflex has several characteristics that make it amenable to cellular studies. The motor neurons and sensory neurons that subserve the reflex are identifiable (Ghysen, 1980; Green, 1981). Extracellular recordings from the leg nerves and sensory neurons are feasible (Wolbarsht and Dethier, 1958; Ikeda and Kaplan, 1970). Thus, investigation of the cleaning reflex allows, in principle, combining the neurogenetic dissection of learning with an analysis of plasticity at the cellular level.

\footnotetext{
Received May 11, 1989; revised July 28, 1989; accepted July 31, 1989.

This study was supported in part by the US-Israel Binational Science Foundation, Jerusalem, and by the US-Israel Binational Agricultural Research and Development Fund (BARD).

Correspondence should be addressed to Yadin Dudai at the above address

a Present address: Department of Anatomy and Neurobiology, Washington University School of Medicine, 660 South Euclid Avenue, St. Louis, MO 63110.

Copyright (C) 1990 Society for Neuroscience $0270-6474 / 90 / 100491-09 \$ 02.00 / 0$
}

In this paper, we report studies on the physiological responses to mechanical stimulation of the sensory neuron that innervates the anteronotopleural (ANP) bristle. Stimulation of this bristle can elicit the cleaning reflex. We have studied 2 experiencedependent modifications of the sensory response: the decay of the response to a sustained stimulus (i.e., adaptation) and the decrement in the response due to repetitive stimulation (i.e., sensory fatigue). These processes were examined in wild-type flies and in the 2 memory mutants that have defects in the cAMP cascade. Our results show that both mutations alter sensory fatigue but do not affect sensory adaptation.

\section{Materials and Methods}

Flies. Canton-S (CS) flies were used as wild-type. The mutants rut and $d n c$ are on a CS genetic background and were kept in homozygous stocks. Olfactory classical conditioning tests (Tully and Quinn, 1985), as well as cAMP PDE (Byers et al., 1981) and adenylate cyclase assays (Dudai et al., 1983), were performed periodically on the flies to detect possible stock contaminations or accumulation of genetic modifiers. Flies carrying the deficiency Df(1)KA9 (Craymer and Roy, 1980) were kept in a stock with the balancer chromosome FM7c. All flies were cultured under standard conditions (Lewis, 1960). Female flies (4-8 d old) were used for experiments. Fly stocks were kept at $24 \pm 1{ }^{\circ} \mathrm{C}, 50-70 \%$ humidity, $12 \mathrm{hr}$ light-12 hr dark cycles. Experiments were performed at $23 \pm 2^{\circ} \mathrm{C}, 50-70 \%$ humidity.

Preparation of flies. Experiments were performed on headless flies. Decapitation of cold-anesthetized flies was performed with iridectomy scissors. Flies were allowed to recover from trauma for $1 \mathrm{hr}$ inside a $35-\mathrm{mm}$-diameter petri dish containing a wet piece of cotton. Only flies that were able to stand correctly on all their legs and to move in response to tactile stimuli applied with forcep tips to the abdomen were used for experiments.

Electrophysiological recordings. A $10 \mathrm{M} \Omega$ impedance metal microelectrode (25-10-1, FHC, New Brunswick) was introduced in the thorax through the scutoscutellar suture. The microelectrode was held by a micromanipulator (M2, Narashige, Japan), and the fly was suspended in the air. For recording, the ANP bristle was cut in the middle with iridectomy scissors, and the open end was introduced into a glass electrode (10-20 $\mu \mathrm{m}$ tip diameter) filled with Drosophila Ringer's (in mM: $130 \mathrm{NaCl}, 2 \mathrm{KCl}, 2 \mathrm{CaCl}_{2}, 5 \mathrm{MgCl}_{2}, 10$ HEPES, pH 7.0). The electrode was held by an electrode holder mounted on a hydraulic micromanipulator (MO-102R, Narashige, Japan). The perspex electrode holder had a piezoelectric crystal (PZT-5H, Vernitron Piezoelectric Division, Bedford, $\mathrm{OH}$ ) that made it possible to move the electrode by application of a square pulse of voltage to the crystal. The pulse was applied by a pulse generator (Grass S88, Quincy, MA) through a stimulus isolating unit (SIU, Grass, Quincy, MA). A 2 M 2 resistor was connected in series with the crystal to avoid ringing (Corey and Hudspeth, 1980). In this configuration, in response to a $25 \mathrm{~V}$ square pulse, the tip of the electrode moved $40 \mu \mathrm{m}$ with a time constant of $5 \mathrm{msec}$. This movement deflected the hair $30 \pm 5^{\circ}$ from its resting position. The direction of the movement was changed by rotation of the electrode holder. The response to deflections of the hair was recorded with a differential amplifier (DAM50, WPI, NJ) and stored on a IBM PC computer with a Computerscope Phy System (RC electronics, Santa Barbara, CA). A sampling rate of 
Figure 1. The anteronotopleural (ANP) bristle and its directional selectivity. The ANP bristle is delimited by a box on the schematic drawing of a decapitated fly and is shown below in an expanded scale. The broken line shows the planc of scctioning used to disconnect the sensory neuron from the CNS in some experiments. The recordings depicted on the right-hand side correspond to each of the 4 directions indicated by Roman numerals in the expanded scale drawing of the ANP bristle: $I$, deflection towards the body wall and back to resting position; $I I$, deflection away from the body wall and back; $I I I$, deflection parallel to the body wall in the ventral direction and back; $I V$, deflection parallel to the body wall in the dorsal direction and back to resting position. The large excursions in the traces are artifacts from the electrical pulse applied to the piezoelectric crystal.
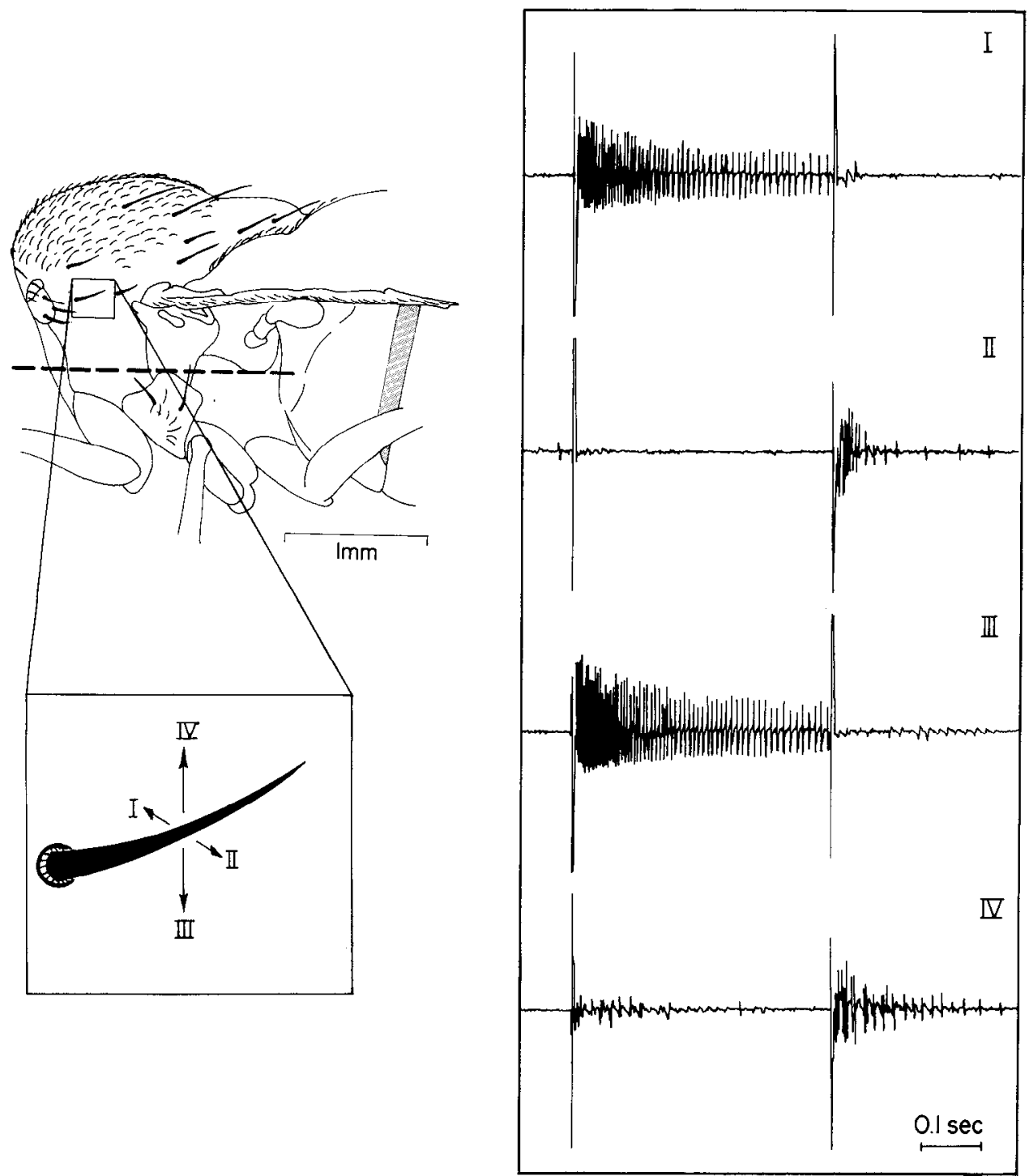

$8.2 \mathrm{kHz}$ was used. Rate meter histograms were obtained for each recording, the bin duration depending on the experimental conditions.

Injection of drugs. Pressure injections of Drosophila Ringer's, $1 \mathrm{~mm}$ 3-isobutyl-1-methylxanthine (IBMX, Sigma) in Ringer's, or $1 \mathrm{mM}$ Ro201724 (Hoffman-La Roche) in Ringer's, were performed with glass capillaries introduced through the scutoscutellar suture. Solution was injected until the abdomen became visibly dilated. The volume thus injected was ca. 0.3-0.4 $\mu \mathrm{l}$.

Mechanical stimulation of the dorsocentral microchaetae simultaneous with electrophysiological recordings. Mechanical stimulation of the dorsocentral microchaetae was performed using an air puff delivery system (Corfas and Dudai, 1989a). Air was delivered via a capillary (50 $\mu \mathrm{m}$ tip diameter), situated at a distance of approximately $0.5 \mathrm{~cm}$ from the fly, and directed to the dorsocentral area of the notum. The capillary was connected to a positive-pressure source (from a central pressure pump), delivering air regulated to a flow of $63 \mathrm{ml} / \mathrm{sec}$ by a flowmeter (Flowrator, Lab-Crest, Warminster, PA). The direction of flow was regulated by 3-way air valves (1-22-900, General Valve, Fairfield, NJ). The valves were controlled by a pulse generator (Grass S88, Quincy, MA) via an electronic interface which converted the square pulse to a biphasic square pulse. The pressured air was delivered in 3 puff trains (1 sec train $/ 3 \mathrm{sec}$ ) of $20 \mathrm{puffs} / \mathrm{sec}, 20 \mathrm{msec}$ puff duration. This experiment was performed on flies fixed by embedding all legs in modeling clay to avoid displacement of the flies by the pressured air stimulus.

Data analysis. Each experiment was performed on 11-19 flies. Unless otherwise indicated, data are presented as means \pm SEM. Two-tailed Student's $t$-test was used for determination of statistical significance of data consisting of 2 sets. Analysis of variance (ANOVA) followed by the Bonferroni $t$-test (overall $\alpha=0.05$ ) were used for determination of statistical significance when more than 2 data sets were compared. All statistical analyses were performed with SAS software (SAS Institute Inc., Cary, NC).

\section{Results}

The ANP neuron responds to deflection of the bristle with a burst of action potentials

The ANP bristle, as the other macrochaetae that cover the notum of $D$. melanogaster, is a Type I mechanosensillum (French, 1988), innervated by a bipolar sensory neuron. This bristle is located on the side of the notum immediately behind the prescutal suture and is directed posteriorly (Fig. 1). The dendrite, containing a modified ciliary structure, is attached to the base of the hair (Burg and Wu, 1986). Upon deflection of the bristle towards the body wall, the ANP neuron response recorded extracellularly is characterized by a negative receptor potential and a burst of positive action potentials. This is similar to the response of some other dipteran sensory neurons (Wolbarsht and Dethier, 1958). The neuron shows directional selectivity in its response (Fig. 1). When deflected towards the body wall, the 


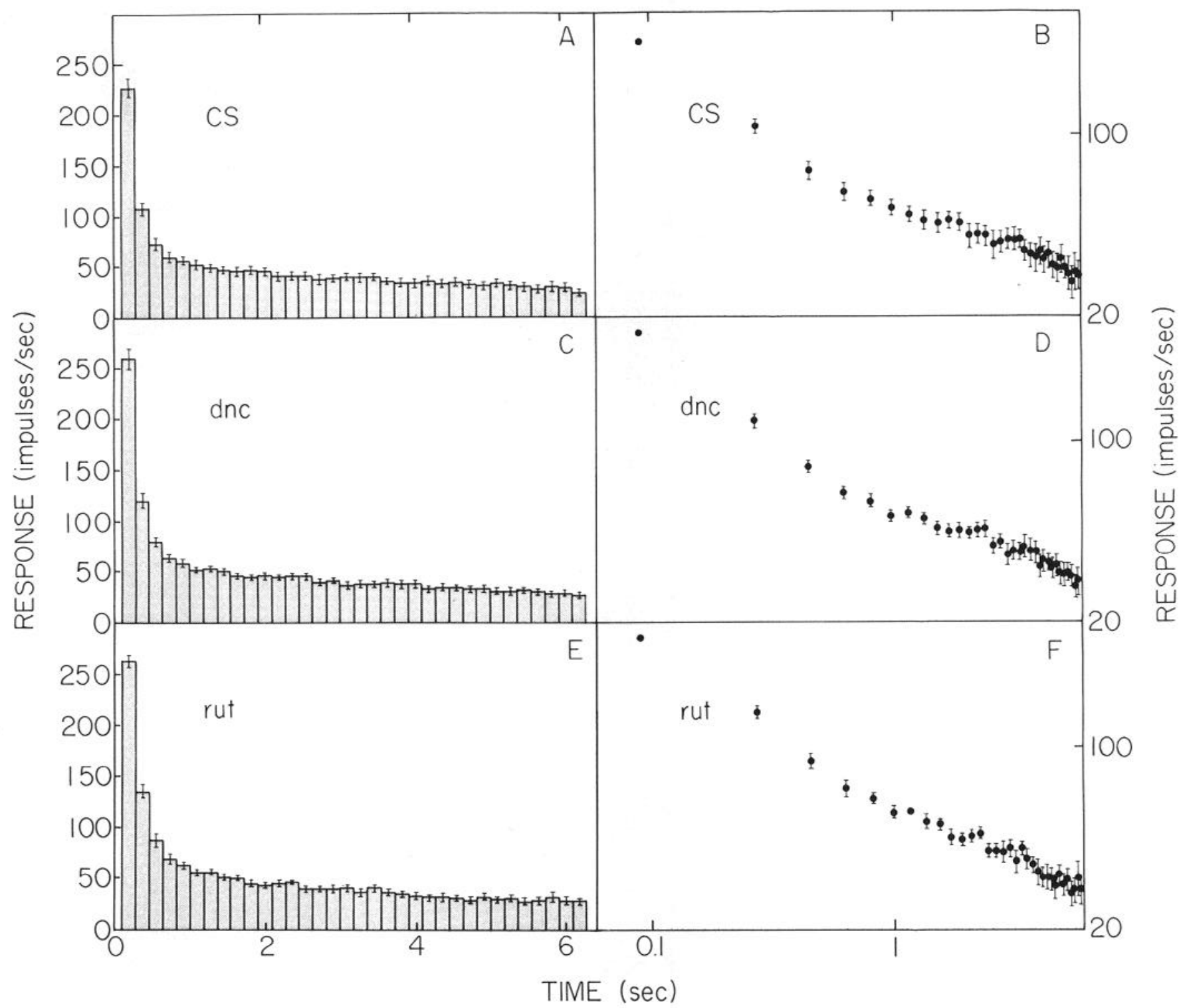

Figure 2. Adaptation of the ANP neuron in response to a $6 \mathrm{sec}$ deflection. Bristles of wild-type $\operatorname{CS}(A, B), d n c(C, D)$, and rut $(E, F)$ flies were stimulated once and the firing frequency plotted (mean \pm SEM) in a linear $(A, C, E)$ and a double $\log$ scale $(B, E, F)$. The number of spikes fired during the duration of the stimulus did not differ significantly among genotypes ( $n=11$ for each genotype).

cell responds with a burst of action potentials that lasts as long as the stimulus is maintained. The same type of response is obtained when the hair is moved from the resting position ventrally in a plane parallel to the body surface. When deflection is away from the body wall or dorsally in a plane parallel to the body surface, the action potentials are fired only during the return to resting position.

Since the deflection of the bristle towards the body wall produces a prominent and readily quantifiable response, we focused our study on the response to this stimulus.

\section{Adaptation of the ANP neuron in response to a sustained deflection towards the body wall}

To characterize the response of the ANP neuron and its adaptation, we recorded the response of the neuron to a 6 sec sustained deflection towards the body wall. We found that the ANP neuron is a slowly adapting mechanosensory neuron. The firing frequency of wild-type (CS) neurons immediately (<10 msec) after deflection was high (350-200 Hz), and declined to a lower rate $(20-10 \mathrm{~Hz})$, which was maintained as long as the stimulus lasted (Fig. 2A). In $d n c$ and rut, the neurons adapted normally (Fig. 2, $C, E$ ). However, an appreciable percentage of $d n c$ flies (approx. 35\%) showed a reduced response to the first mechanical stimulation, i.e., they fired a short burst of action potentials and remained silent during the rest of the stimulus period. In contrast, only less than $3 \%$ of CS and rut flies showed reduced response to the first stimulus. In all cases the flies with reduced initial response were discarded in this study.

Adaptation in this system appears similar to that described in several other invertebrate and vertebrate sensory neurons, whose firing rate in response to a sustained stimulus follows a power law (Chapman and Smith, 1963; Thorson and Biederman-Thorson, 1974; Watts and French, 1985). Thus, the decay of the firing rate can be described by the equation

$$
R=C t^{-k}
$$




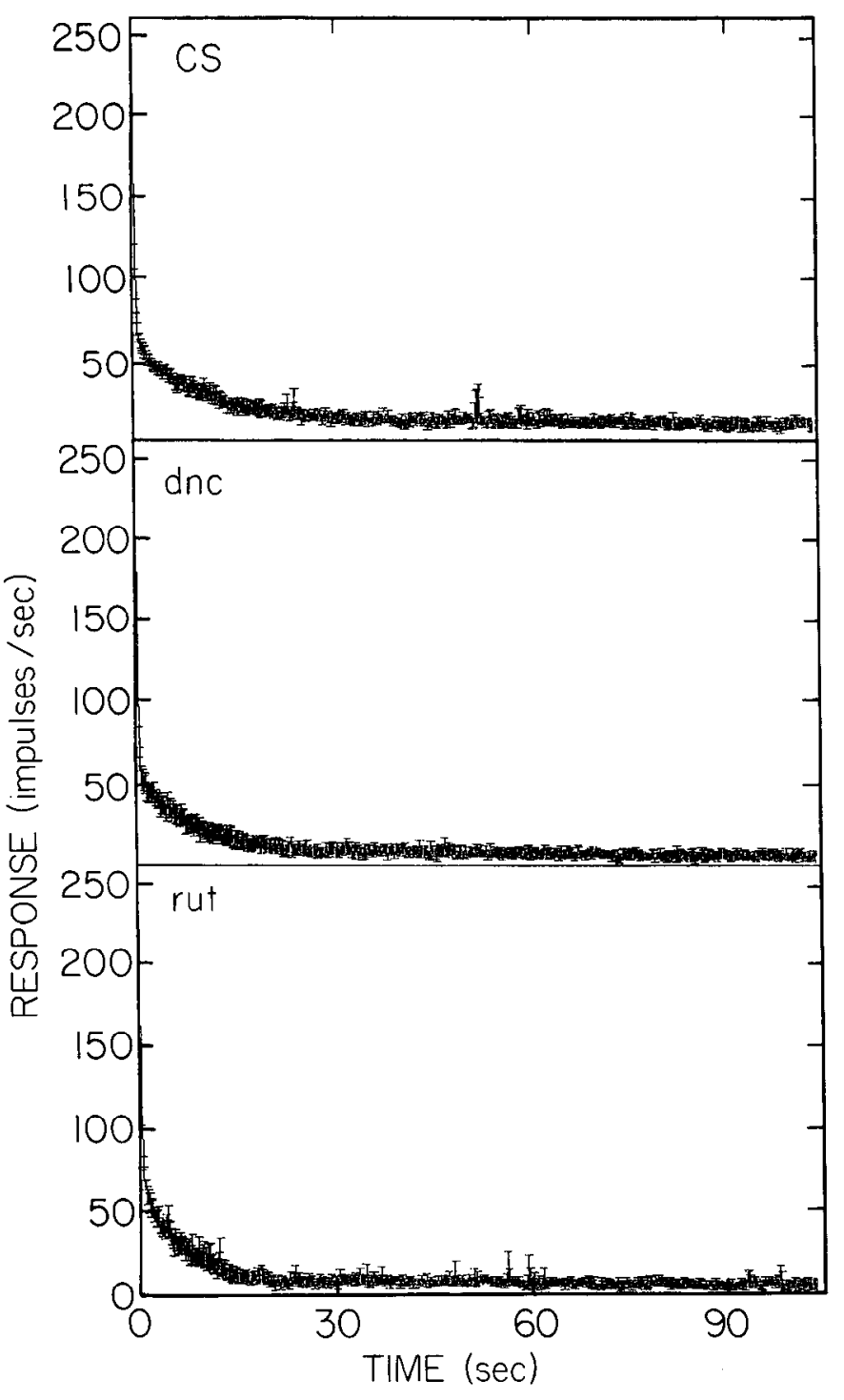

Figure 3. Adaptation of the ANP neuron in response to a $100 \mathrm{sec}$ deflection. Bristles of wild-type CS, $d n c$, and rut flies were stimulated once and the firing frequency plotted (mean \pm SEM) as a function of time. The number of spikes fired during the duration of the stimulus did not differ significantly among genotypes $(n=11$, for each genotype).

where $R$ is the response of the cell measured in firing frequency, $C$ is the firing frequency after $1 \mathrm{sec}, t$ is time after onset of the stimulus, and $k$ is a fractional exponent of the time (Chapman and Smith, 1963; Thorson and Biederman-Thorson, 1974).

To make a quantitative comparison of the adaptation of sensory neurons of wild-type and memory mutant flies, we fitted the data of the response as a function of time to a power-law function and calculated $k$ (by least-squares criteria). There were no significant differences in the values of $C$ and $k$ for the different genotypes (CS: $C=52.16 \pm 3.74, k=0.51 \pm 0.034$; dnc: $C=$ $51.42 \pm 2.73, k=0.49 \pm 0.034 ;$ rut: $C=55.83 \pm 2.29, k=$ $0.53 \pm 0.024)$. These results indicate that memory mutations do not affect the adaptation of the ANP sensory neuron in response to $6 \mathrm{sec}$ sustained deflection.

When a $100 \mathrm{sec}$ stimulus was used, the firing frequency decreased rapidly during the first few seconds (see above), but the cells continued to respond as long as the bristle was deflected

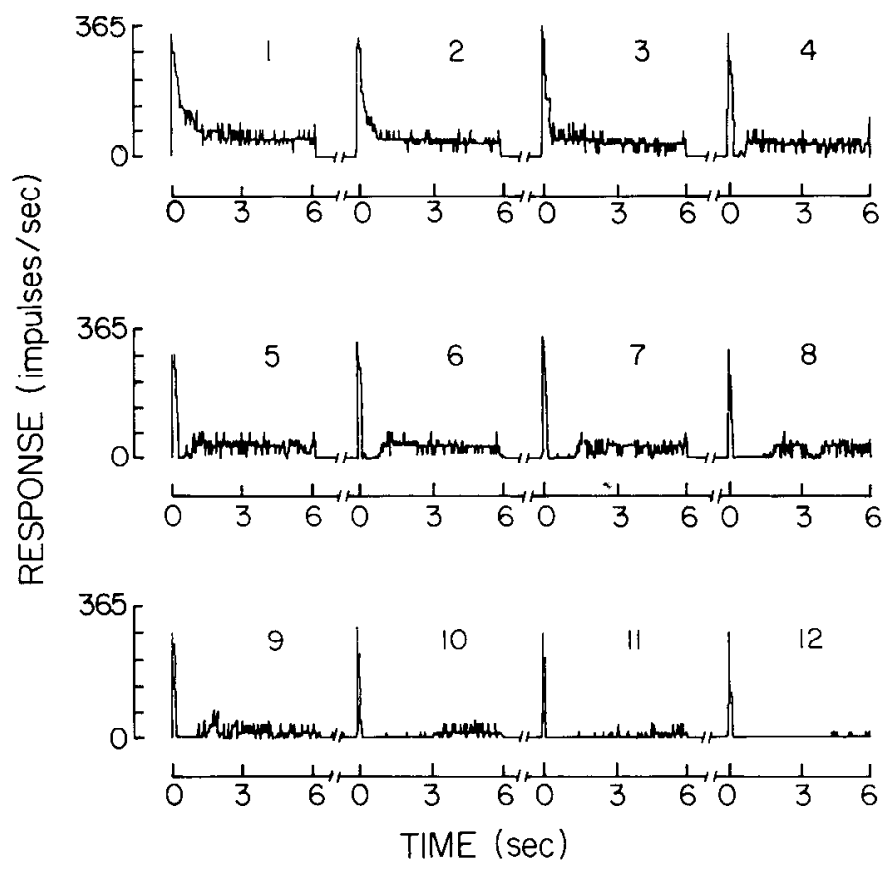

Figure 4. Adaptation and fatigue of a representative wild-type neuron upon 12 consecutive stimuli ( 6 and $20 \mathrm{sec}$ ISI). The stimulus number is shown on each trace.

(Fig. 3). After $90 \mathrm{sec}$ of stimulation, the firing frequencies of the neurons in all genotypes were as expected from equation 1.

\section{Effect of repetitive brief stimulations on the mechanosensory response}

In our behavioral studies on the cleaning reflex (Corfas and Dudai, 1989a), we have used repetitive mechanical stimulation of the thoracic bristles to study the plasticity of the behavioral response. In the present study, we followed the response of the ANP neuron to repetitive mechanical stimulation in order to investigate the plasticity at the cellular level. In these experiments, the number of spikes fired in response to each stimulus was determined and normalized with respect to the number of spikes fired in response to the first stimulus. Each fly was subjected to a single set of stimuli.

The following stimulation protocols were used in the present study: (1) Stimuli of $6 \mathrm{sec}$ with $20 \mathrm{sec}$ interstimulus interval (ISI). This protocol was used because a 6 sec stimulus was employed in our studies on adaptation (see above). (2) Stimuli of $400 \mathrm{msec}$ with $5 \mathrm{sec}$ ISI. This protocol is similiar to that used in our previous studies on the habituation of the cleaning reflex (Corfas and Dudai, 1989a). (3) Stimuli of $400 \mathrm{msec}$ with 1, 2, or $20 \mathrm{sec}$ ISI. These protocols were used to study the dependence of decrement of sensory response on the rate of stimulation (see below).

Repetitive stimulation of ANP neurons of wild-type flies (6 $\mathrm{sec}$ stimuli, $20 \mathrm{sec}$ ISI) led to a progressive decrement in burst duration and in firing frequency. In many cases, neurons which responded to the first stimulus with a long burst, responded after several stimuli with a short hurst and remained silent for the rest of the stimulus (Fig. 4).

When the responses to consecutive stimuli were plotted on a double log scale (Fig. 5A), the changes in adaptation kinetics became even more evident. After several stimuli, the firing rate 


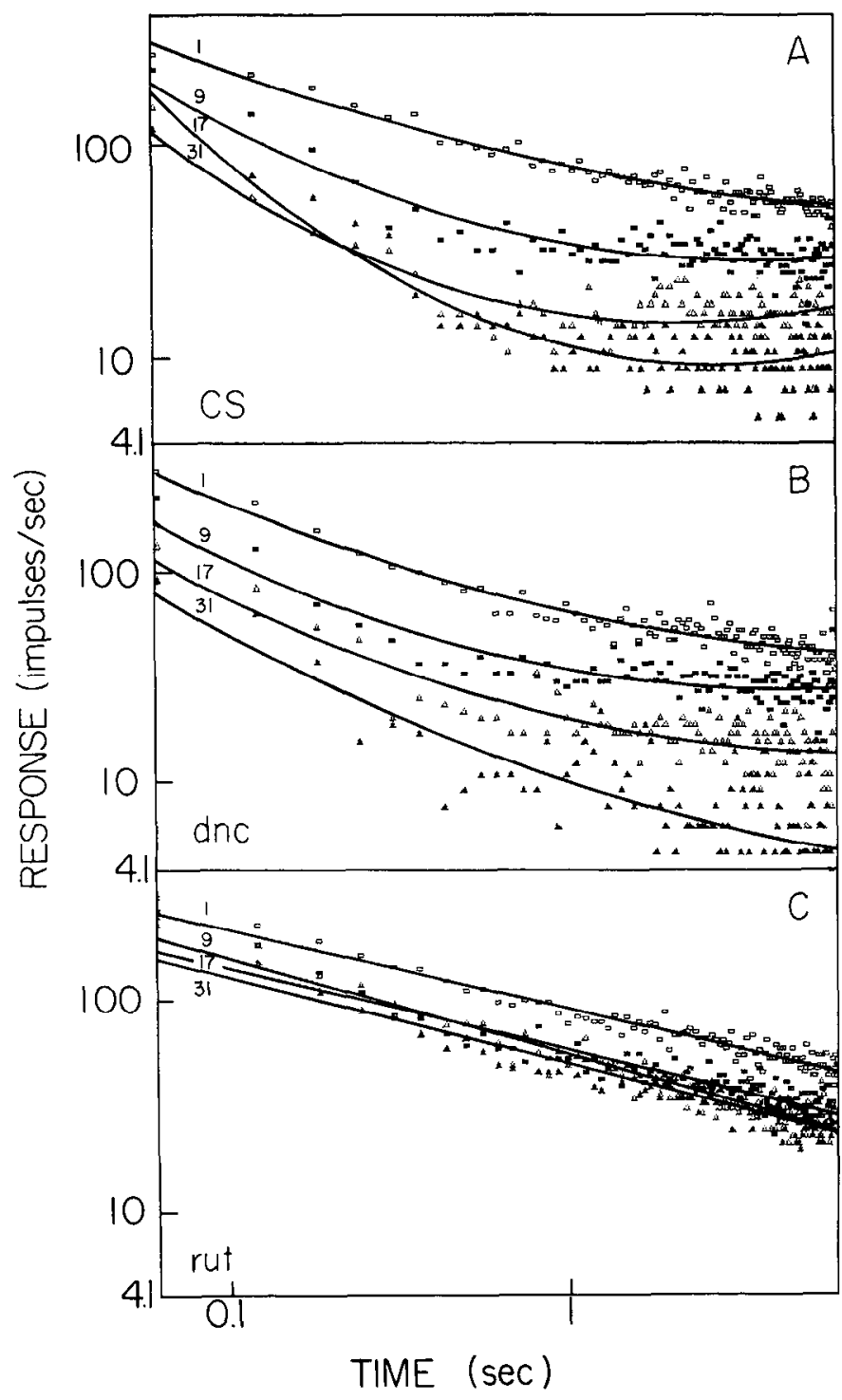

Figure 5. Adaptation and fatigue in response to $6 \mathrm{sec}$ stimuli with 20 sec ISI. Bristles of wild-type CS $(A)$, dnc $(B)$, and rut $(C)$ flies were subjected to 31 consecutive stimuli ( $n=12$ for each genotype). The mean responses to the $1,9,17$, and 31 stimuli were plotted on double log scale. The points were fitted to a second-order polynomial equation (full lines) to assist in visualizing the data.

no longer followed a linear power-law function. The firing frequency decayed more rapidly and to lower values than in response to the first stimulus (Fig. 5). This change in sensory response due to repetitive stimulation has been reported to occur in different mechanusensory systems (Roberts and Hayes, 1977; Bush and Pasztor, 1983; Watts and French, 1985; Baumann et al., 1987) and is called sensory fatigue (Monnier, 1975).

Fatigue was found to be a function of stimulus duration. With identical stimulation rates, longer stimuli resulted in rapid fatigue, whereas a very brief stimulus resulted in very little fatigue (Fig. 6).

The change in sensory response due to repetitive stimulation is independent of CNS feedback

To determine if the process of sensory fatigue is a cell autonomous process or, alternatively, is due to CNS feedback inhi-

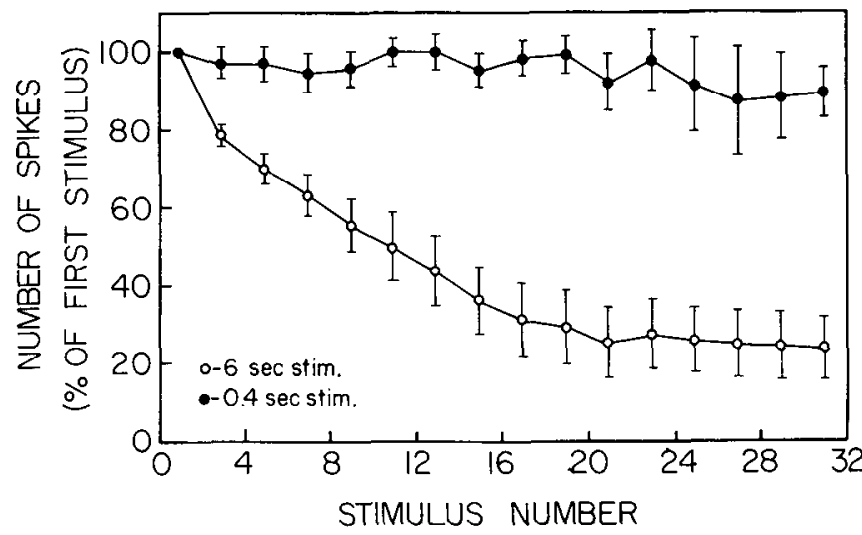

Figure 6. Stimulus-duration dependence in fatigue of wild-type neurons. Bristles were deflected for $400 \mathrm{msec}$ at $5 \mathrm{sec}$ ISI and for $6 \mathrm{sec}$ at $20 \mathrm{sec}$ ISI ( $n=11$ for each rate of stimulation). The number of spikes fired in response to each stimulus was normalized to the number of spikcs fircd in response to the first stimulus in each fly.

bition, we monitored the fatigue of ANP neurons isolated from the CNS. The flies were cut in the horizontal plane, at the level of the pleural cleft (Fig. 1). In this way, the notum was completely separated from the CNS. These flies showed normal sensory fatigue in the ANP neuron in response to $6 \mathrm{sec}$ stimuli presented with $20 \mathrm{sec}$ ISI (data not shown).

\section{Sensory neurons do not recover from fatigue with a dishabituating stimulus}

In our previous studies on the behavioral habituation of the cleaning reflex (Corfas and Dudai, 1989a), we observed that behavioral response can be restored immediately after habituation by application of a dishabituating stimulus to the dorsocentral microchaetae. To investigate whether sensory response is also restored to fatigued neurons by a dishabituating stimulus, the following experiment was performed. ANP neurons from wildtype flies were subjected to repetitive mechanical stimulation (6 sec stimuli, $20 \mathrm{sec}$ ISI) for 31 stimuli. This stimulation regime resulted in fatigue (see above). Immediately after the last stimulus, the dishabituating stimulus was applied to the dorsocentral area of the notum. Following the dishabituating stimulus, the ANP neuron was stimulated once more and its response recorded. The responses of the ANP neuron immediately before and after application of the dishabituating stimulus were not significantly different (data not shown).

\section{The ANP sensory neuron in rut fatigues less than the ANP neuron in wild-type and dnc}

Since sensory fatigue is an experience-dependent modification of the sensory response, we decided to test whether learning mutations affect it. As shown in Figures $5 B$ and $7 B$, upon repetitive stimulation (6 sec stimuli, $20 \mathrm{sec}$ ISI), sensory fatigue of the ANP neuron of $d n c$ was indistinguishable from that in the wild-type. However, the response of rut neurons was more resistant to repetitive stimulation, and after a small decrement that resulted from the first few stimuli, there was no further significant change in the response (Figs. $5 C, 7 A$ ).

To investigate whether the abnormally slow sensory fatigue in rut neurons was indeed caused by the rut mutation, we tested if this physiological abnormality maps to the rut locus in the $\mathrm{X}$ chromosome. This was performed by testing female flies with one $\mathrm{X}$ chromosome carrying either the rut or the $r u t^{+}$(wild- 


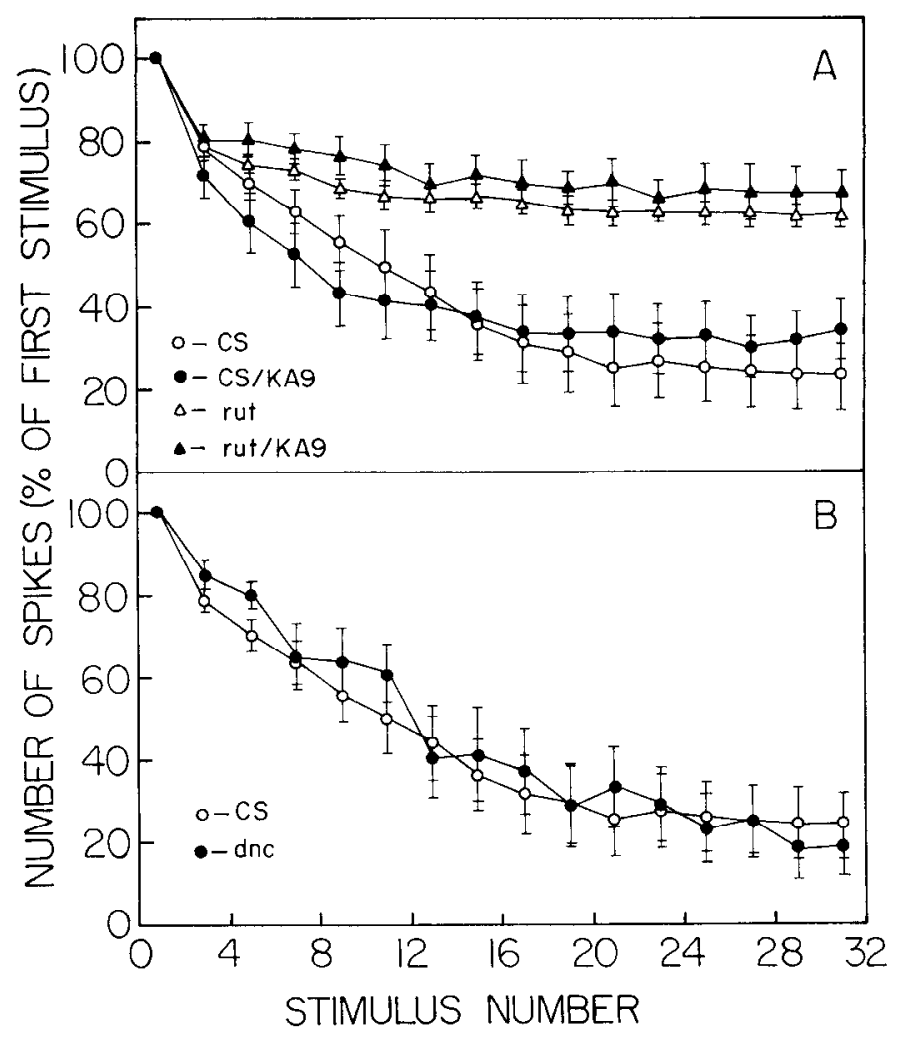

Figure 7. Evidence that the effect on sensory fatigue maps to the rut region. $A, \operatorname{CS}(n=11)$, rut $(n=19), \mathrm{CS} / \mathrm{KA} 9(n=11)$, and $r u t / \mathrm{KA} 9(n$ $=13$ ) neurons were subjected to repetitive stimulation with $6 \mathrm{sec}$ at 20 sec ISI. The ANOVA test for the response to the last stimulus revealed differences between the samples ( $p>F=0.0001)$. The Bonferroni $t$-test showed that the flies carrying the rut mutation either in an homozygous (rut) or hemizygous (rut/KA9) state were significantly different from wild-type. $B, d n c(n=11)$ neurons subjected to the same stimulation protocol behaved like CS $(n=11)$ neurons.

type) gene, and the other $\mathrm{X}$ chromosome with a deficiency that uncovers the rut locus. The deficiency Df(1)KA9 (Craymer and Roy, 1980), missing polytenic bands 12E1-13Al (R. Kreber, personal communication), was used, since it covers the rut locus (12F5-6 to 13A1; Livingstone et al., 1984; Dudai et al., 1985). Mechanosensory neurons of flies with the genotype $r u t / \mathrm{KA} 9$ showed the same behavior as rut/rut flies, whereas neurons of $r u t^{+} /$KA9 flies behaved like wild-type (Fig. $7 A$ ). This allows us to state that the mutation responsible for the abnormally slow fatigue maps to the same small chromosomal region as rut, and is possibly rut.

\section{dnc fatigues more rapidly than $C S$ and rut neurons}

Under the above-mentioned conditions $d n c$ neurons fatigued at a normal rate (Fig. $7 B$ ). However, under other conditions in which neither CS nor rut demonstrated fatigue (400 msec stimuli, $5 \mathrm{sec}$ ISI), $d n c$ fatigued noticeably (Fig. 8). Although the kinetics of fatigue in rut and $d n c$ differed from normal and from each other, the neurons from all genotypes recovered from fatigue with a similar rate after repetitive mechanical stimulation under this stimulation protocol (Fig. 8).

The dependence of sensory fatigue on the stimulation rate is affected by the dnc mutation

In CS and rut flies, sensory fatigue was significantly more dependent on the rate of stimulation than in $d n c$ flies (Fig. 9).

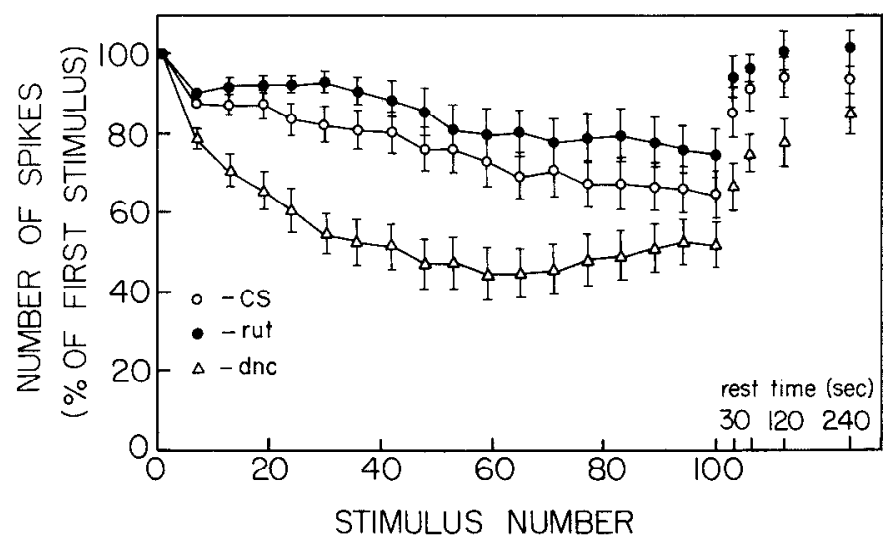

Figure 8. Fatigue in response to repetitive stimulation with $400 \mathrm{msec}$ stimuli at $5 \mathrm{sec}$ ISI. Wild-type CS, rut, and $d n c$ neurons were subjected to 100 consecutive stimuli and retested $30 \mathrm{sec}$ and 1,2 , and 4 min after the last stimulus to quantify the recovery of response $(n=12$ for each genotype). The ANOVA test for the response to the 30th stimulus revealed differences between the samples $(p>F=0.0001)$; the Bonferroni $t$-test showed that the response of $d n c$ neurons was significantly different from the response of both CS and rut.

Thus, in both rut and CS flies after 31 consecutive stimuli, the responses of neurons stimulated with $400 \mathrm{msec}$ stimuli at 1, 2, or $5 \mathrm{sec}$ ISI were significantly different from each other. In contrast, in $d n c$ the response after stimulation at $2 \mathrm{sec}$ ISI was not significantly different from the responses after stimulation at $1 \mathrm{sec}$ ISI.

\section{Inhibitors of CAMP PDE affect sensory fatigue in CS}

Since $r u t$ and $d n c$ are cAMP cascade mutants (reviewed in Dudai, 1988), their abnormal sensory fatigue suggests that cAMP metabolism plays a role in this process. To test this hypothesis we studied the effect of inhibitors of cAMP PDE which increases cAMP levels, on sensory fatigue. Drugs were injected as detailed under Materials and Methods, and recordings were performed starting $5 \mathrm{~min}$ after injection. As seen in Figure 10, both PDE inhibitors tested, IBMX and Ro20-1724, affected wild-type but not rut neurons. Under the conditions employed $(400 \mathrm{msec}$ stimuli, $5 \mathrm{sec}$ ISI), CS and rut neurons responded similarly to repetitive stimulation in the absence of drug treatment (Fig. $10 A$ ) but behaved differently after drug injection (Fig. 10, $B, C$ ). Upon drug treatment, the sensory neurons in CS fatigued more rapidly than normal, resembling $d n c$. This suggests that the genetic defect in cAMP PDE in $d n c$ is responsible for the observed altered fatigue and implicates cAMP in the process underlying fatigue in mechanosensory neurons.

\section{Sensory fatigue following deflection of the bristle away from the body wall}

In our previous studies on the behavioral habituation of the cleaning reflex (Corfas and Dudai, 1989a), we monitored the response of the flies to air puffs that deflected the thoracic bristles away from the body wall. In contrast, the stimulus used in the clcctrophysiological cxpcrimcnts described in this paper was deflection towards the body wall. This, as explained above, was due to the more prominent and quantifiable response obtained with deflection towards the body wall. However, to determine whether sensory fatigue plays a role in behavioral habituation, it is pertinent to study also the response of the ANP neuron to stimuli which are more similar to those used in the previous 


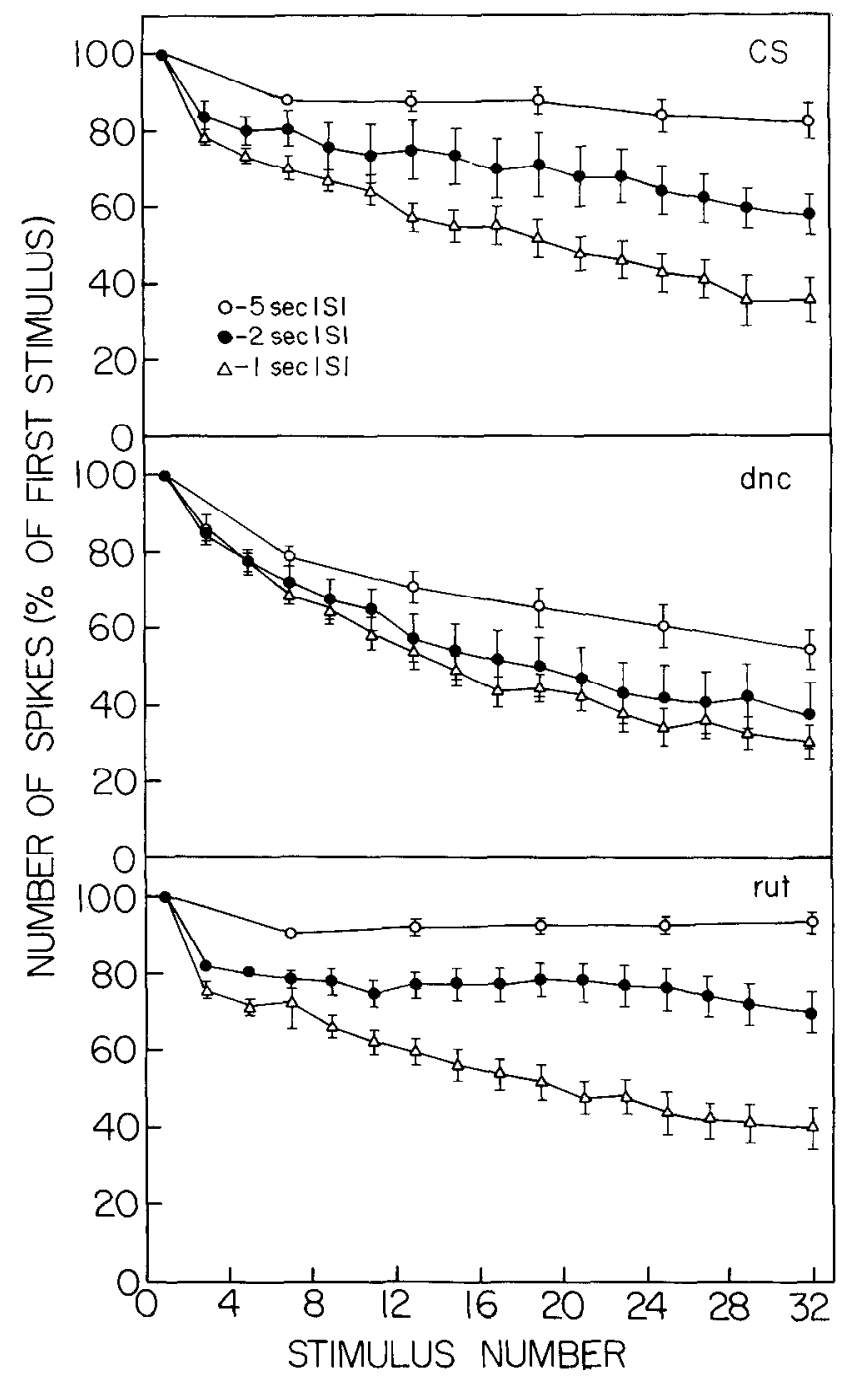

Figure 9. Stimulation-rate dependence of fatigue and the effect of mcmory mutations. Bristles of wild-type CS $(A), d n c(B)$, and rut $(C)$ were deflected for $400 \mathrm{msec}$, at $5 \mathrm{sec}$ ISI, $2 \mathrm{sec}$ ISI, and $1 \mathrm{sec}$ ISI $(n=$ 11 for each genotype and stimulation rate). The number of spikes fired in response to each stimulus was normalized to the number of spikes fired in response to the first stimulus in each fly. The responses to the last stimulus at different frequencies of stimulation are significantly different in rut and CS flies (by ANOVA followed by the Bonferroni $t$-test). The response to the last stimulus is not significantly different in $d n c$ neurons stimulated with 2 sec ISI when compared with neurons stimulated with either 5 or $1 \mathrm{sec}$ ISI (by $t$-test). The responses of $d n c$ neurons stimulated with $5 \mathrm{sec}$ ISI are significantly different from those stimulated with $1 \mathrm{sec}$ ISI $(p<0.006, t$-test $)$.

behavioral studies. Therefore, we monitored the response of sensory neurons to repetitive deflection away from the body wall, using a stimulation regimen similar to that used in the behavioral studies. In this experiment, the stimulation protocol consisted in $400 \mathrm{msec}$ deflections away from the body wall (5 sec ISI). We quantified the number of spikes fired during the first $50 \mathrm{msec}$ after the offset of the deflection, since this was the time span within which most of the spikes were elicited (Fig. 1). The decrement in response to repetitive deflections away from the body wall was similar to that due to repetitive deflections towards the body wall (Figs. 8, 11). This suggests that sensory fatigue may be independent of stimulus direction and initial burst duration.

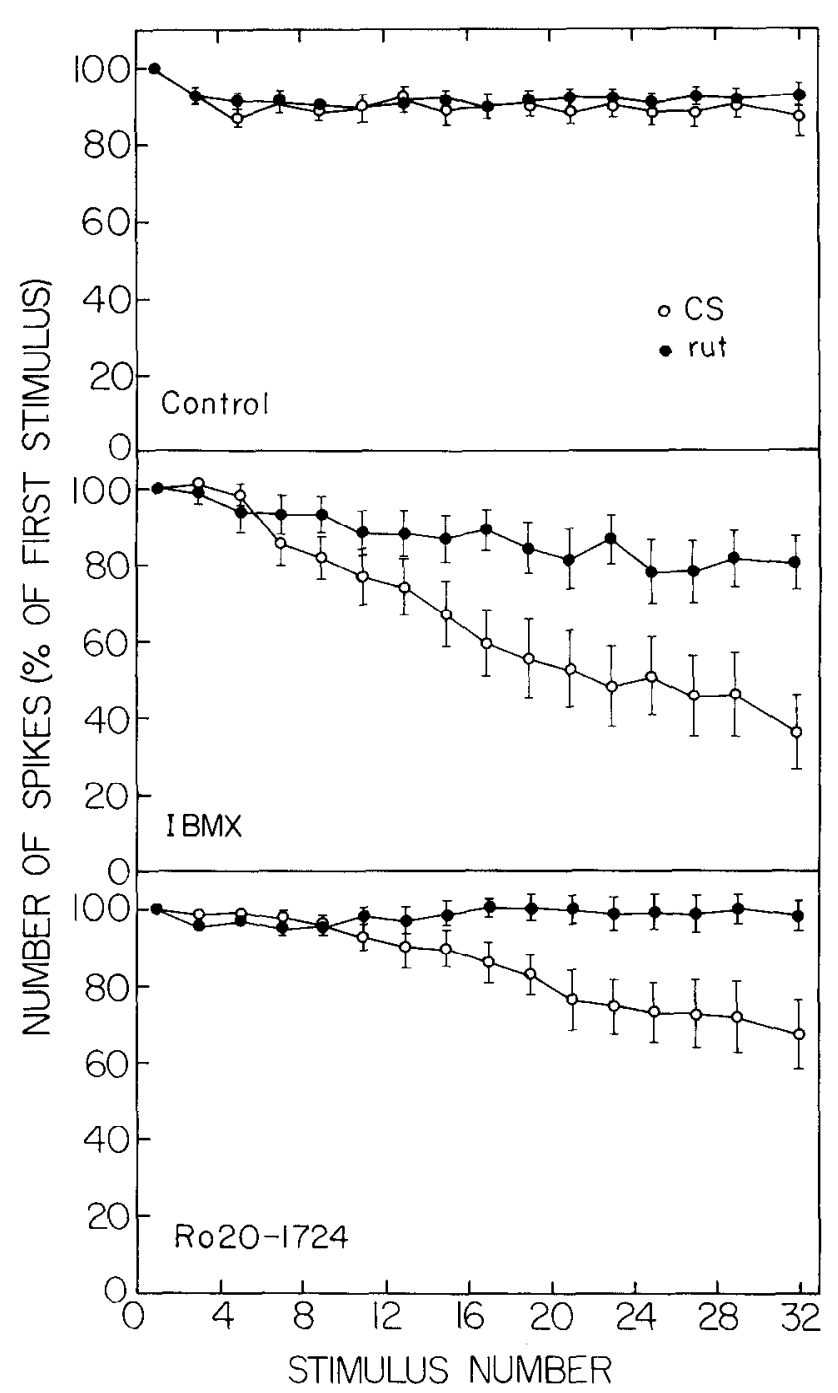

Figure 10. Effect of cAMP phosphodiesterase inhibitors on sensory fatigue. Wild-type CS and rut neurons were tested for fatigue in response to $400 \mathrm{msec}$ stimuli, at $5 \mathrm{sec}$ ISI, $5 \mathrm{~min}$ after injection of Ringer's (Control: CS, $n=11 ;$ rut, $n=11$ ), $1 \mathrm{mM}$ IBMX (CS, $n=16 ; r u t, n=$ 17), or $1 \mathrm{~mm}$ Ro20-1724 (CS, $n=11 ; r u t, n=11)$. The fatigue in response to repetitive stimulation following drug treatment differs between the genotypes (IBMX, $p<0.002$; Ro20-1724, $p<0.01$ in the last stimulus).

\section{Discussion}

\section{Response of the ANP neuron to mechanical stimulation}

The ANP neuron adapts to a sustained stimulus in a manner similar to that displayed by other invertebrate and vertebrate mechanosensory neurons (Thorson and Biederman-Thorson, 1974; Watts and French, 1985). After repeated stimulation, the response to deflection changes and the neuron adapts more rapidly and with different kinetics. The decline of mechanosensory response to repetitive stimulation was termed sensory fatigue (Monnier, 1975). Fatigue has been described in several mechanosensory systems, including mammals (Baumann et al., 1987), crustacea (Bush and Pasztor, 1983), and amphibians (Roberts and Hayes, 1977; Watts and French, 1985). Different types of mechanosensory neurons in the same organism may differ in their fatigue characteristics (Watts and French, 1985). Sensory fatigue has not been profoundly studied, and its mechanism is 


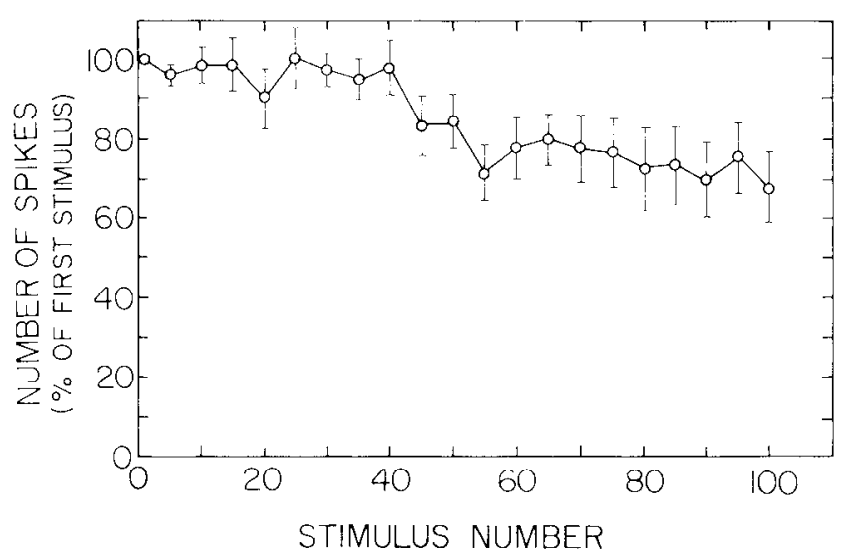

Figure 11. Response and fatigue of the ANP neuron to deflection away from the body wall and back to rest. CS sensory neurons $(n=11)$ were deflected for $400 \mathrm{msec}$ away from the body wall at $5 \mathrm{sec}$ ISI. The response was recorded, and the number of spikes fired during the first $50 \mathrm{msec}$ after the offset of the stimuli was quantified and normalized to the response to the first stimulus.

yet unknown. A possible explanation for the decline in sensory response due to repetilive stimulation is that it is caused by an incomplete recovery from adaptation from one stimulus to the other. This would imply that sensory fatigue is not different from adaptation but is a result of the regimen of stimulation. A sccond possibility is that sensory fatigue is a process which differs from adaptation and that it acts by modifying the molecular mechanisms underlying mechanosensory response or adaptation.

In the present system, sensory fatigue is manifested as a change in the sensory response and in the adaptation kinetics. Moreover, memory mutations which impair the cAMP cascade affect sensory fatigue but not sensory adaptation. This suggests that adaptation and sensory fatigue are subserved by different mechanisms. Note that so far, adaptation has not been linked to second-messenger cascades, but rather to changes in the mechanical coupling between sensory organ and neuron (Loewenstein and Mendelson, 1965), in mechanotransduction mechanisms (Eatock et al., 1987), and in the spike-generation mechanism (French, 1984). Since the molecular defects of mt and $d n c$ are in the CAMP cascade, we would like to suggest that fatigue can be triggered by the cAMP cascade. Thus, stimulation of the mechanosensory neuron may somehow generate cAMP, which in turn may lead to physiological changes manifested as fatigue, via protein phosphorylation or via direct interaction of CAMP with membrane components such as ion channels (Nestler and Greengard, 1984; Dudai, 1987). The similarity in the rate of recovery from fatigue in all the genotypes suggests that $r u t$ and $d n c$ gene products are not critical in the recovery process.

\section{Effect of drugs that affect the CAMP cascade and of memory mutations on adaptation and fatigue}

The memory mutants rut and $d n c$ do not differ from the wild type in the intensity and adaptation of the rcsponse to the first stimulus, but they do differ in the response to repetitive monotonous stimulation. While rut flies fatigue less rapidly than wild-type, dnc flies fatigue more rapidly. The opposite effects of the mutations on the physiological response parallel the opposite effects that these mutations have on cAMP levels. rut has a defective $\mathrm{Ca} /$ /calmodulin-activated adenylate cyclase and thus has reduced cAMP synthesis. dre has a defective cAMP PDE, and thus has reduced CAMP hydrolysis. The correlation of fatigue with the CAMP levels is supported by results obtained by genetic analysis in the case of mt and by pharmacology in the case of dnc. The effect of rut on sensory fatigue was mapped to the region of the $\mathrm{X}$ chromosome which affects the activity of adenylate cyclase. Systemic injection of PDE inhibitors mimicked the effect of $d n c$ in the wild type, but not in mut, which has an abnormally low cAMP production.

Since memory mutations may affect general fitness, we must consider the possibility that the effects of $d n c$ and rut described in this work are due to reduced fitness. However, we do not think that this is the case. rut increases the "performance" of the cell, while dne affects only sensory fatigue but not the recovery from it. dne has also been shown not to be significantly affected in motor activity and phototaxis, indices of general fitness (Dudai et al., 1976). Iowever, it should be noted that ca. $35 \%$ of dnc neurons behaved like being fatigued already when stimulated for the first time. This might result from ahnormally rapid fatigue induced by stimulation of the neuron during preparation of the flies for the test.

\section{Physiological and behavioral effects of memory mutations}

A drawback of the neurogenetic dissection of learning in Drosophila is the lack of information on the effect of learning mutations on neurons that can potentially contribute to behavioral plasticity. Therefore, interpretation of the effects of Drosophila learning mutations draws from cellular analysis of learning in molluses (reviewed in Tully, 1987, and Dudai, 1988). To the best of our knowledge, this is the first study to address the effect of learning mutations on properties of an identified, single neuron that is capable of subserving a modifiable behavior.

We have been able to show that 2 memory mutations affect the physiology of the mechanosensory neuron that subserves the cleaning reflex. Whereas the mutations do not significantly affect the naive sensory response and its adaptation, they do alter the decrement of response to repetitive stimulation, i.e., sensory fatigue. At this stage we cannot yet determine the contribution of sensory fatigue to the habituation of the cleaning reflex. However, we can already conclude that sensory fatigue does not play a cardinal role in the most prominent effect of $d n c$ and rut on the reflex, which is a marked abbreviation of the memory of habituation. This is because $d n c$ and rut have opposite effects on mechanosensory fatigue but have the same effect on the memory of habituation (Corfas and Dudai, 1989a, b). Furthermore, the cleaning reflex undergoes dishabituation, while fatigue of the ANP neuron is not affected by the stimulation of other neurons. Taken together, our findings imply that even if the abnormal functional properties of the mechanosensory neuron are relevant to learning and memory defects, the relationship is not straightforward, and central mechanisms must be considered.

\section{References}

Baumann, K. I., W. Hamann, and W. H. Yung (1987) Lithium and the excitability of slowly adapting type I (s.a. I) cutaneous mechanoreceptors of the rat. J. Physiol. (Lond.) 390:38P.

Burg, M. G., and C.-F. Wu (1986) Differentiation and central projection of peripheral sensory cells with action potential block in Drosophila mosaics. J. Neurosci. 6: 2968-2970.

Bush, B. M. H., and V. M. Pastor (1983) Adaptation and sensory habituation in primary mechanoreceptive afferent of the lobster oval organ. J. Physiol. (Lond.) 343: 26P-27P. 
Byers, D., R. L. Davis, and J. A. Kiger (1981) Defect in cyclic AMP phosphodiesterase due to the dunce mutations of learning in Drosophila melanogaster. Nature 289: 79-81.

Chapman, K. M., and R. S. Smith (1963) A linear transfer function underlying impulse frequency modulation in cockroach mechanoreceptor. Nature 197: 699-700.

Corey, D. P., and A. J. Hudspeth (1980) Mechanical stimulation and manipulation with piezoelectric bimorph elements. J. Neurosci. Methods 3: 183-202

Corfas, G., and Y. Dudai (1989a) Habituation and dishabituation of a cleaning reflex in normal and mutant Drosophila. J. Neurosci. 9: 56-62.

Corfas, G., and Y. Dudai (1989b) Memory mutations affect an elementary experience-dependent modification of response in an identified sensory neuron of Drosophila. Soc. Neurosci. Abstr. 15: 1117.

Craymer, L., and E. Roy (1980) New mutants: Drosophila melanogaster. Drosophila Inf. Serv. 55: 200-204.

Dudai, Y. (1987) The cAMP cascade in the nervous system: Molecular sites of action and possible relevance to neuronal plasticity. CRC Crit. Rev. Biochem. 22: 221-281.

Dudai, Y. (1988) Neurogenetic dissection of learning and short-term memory in Drosophila. Annu. Rev. Neurosci. 11: 537-563.

Dudai, Y., and S. Zvi (1984) Adenylate cyclase in the Drosophila memory mutant rutabaga displays an altered $\mathrm{Ca}^{2+}$ sensitivity. Neurosci. Lett. 47: 119-124.

Dudai, Y., Y.-N. Jan, D. Byers, W. G. Quinn, and S. Benzer (1976) dunce, a mutant of Drosophila deficient in learning. Proc. Natl. Acad. Sci. USA 73: 1684-1688.

Dudai, Y., A. Uzzan, and S.Zvi (1983) Abnormal activity of adenylate cyclase in the Drosophila memory mutant rutabaga. Neurosci. Lett. 42: 207-212.

Dudai, Y., B. Sher, D. Segal, and Y. Yovell (1985) Defective responsiveness of adenylate cyclase to forskolin in the Drosophila memory mutant rutabaga. J. Neurogenet. 2: 365-380.

Eatock, R. A., D. P. Corey, and A. J. Hudspeth (1987) Adaptation of mechanoelectrical transduction in hair cells of the bullfrog's sacculus. J. Neurosci. 7: 2821-2836.

French, A. S. (1984) Action potential adaptation in the femoral tactile spine of the cockroach, Periplaneta americana. J. Comp. Physiol. A 155: 803-812.

French, A. S. (1988) Transduction mechanisms of mechanosensilla. Annu. Rev. Entomol. 33: 39-58.
Ghysen, A. (1980) The projection of sensory neurons in the central nervous system of Drosophila: Choice of the appropriate pathway. Dev. Biol. 78: 521-541.

Green, S. H. (1981) Segment-specific organization of leg motoneurones is transformed in bithorax mutants of Drosophila. Nature 286: 6567

Ikeda, K., and W. D. Kaplan (1970) Patterned neural activity of a mutant Drosophila melanogaster. Proc. Natl. Acad. Sci. USA 66:765772.

Lewis, E. G. (1960) A new standard food medium. Drosophila Inf Ser. 34: 117-118.

Livingstone, M. S., P. P. Sziber, and W. G. Quinn (1984) Loss of calcium/calmodulin responsiveness in adenylate cyclase of rutabaga a Drosophila learning mutant. Cell 37: 205-215.

Loewenstein, W. R., and M. Mendelson (1965) Components of receptor adaptation in a Pacinian corpuscule. J. Physiol. (Lond.) 177 : 377-397.

Monnier, M. (1975) Functions of the Nervous System. Vol. 3, Sensory Functions and Perception, Elsevier, New York.

Nestler, E. J., and P. Greengard (1984) Protein Phosphorylation in the Nervous System, Wiley, New York.

Power, M. E. (1948) The thoracico-abdominal nervous system of an adult insect. Drosophila melanogaster. J. Comp. Neurol. 88: 347-409.

Roberts, A., and B. P. Hayes (1977) The anatomy and function of 'free' nerve endings in an amphibian skin sensory system. Proc. R. Soc. London [Biol.] 196: 415-429.

Thorson, J., and M. Biederman-Thorson (1974) Distributed relaxation processes in sensory adaptation. Science 183: 161-172.

Tully, T. (1987) Drosophila learning and memory revisited. Trends Neurosci. 10: 330-335.

Tully, T., and W. G. Quinn (1985) Classical conditioning and retention in normal and mutant Drosophila melanogaster. J. Comp. Physiol. 157: 263-277.

Vandervorst, P., and A. Ghysen (1980) Genetic control of sensory connections in Drosophila. Nature 286: 65-67.

Watts, R. E., and A. S. French (1985) Sensory transduction in dorsal cutaneous mechanoreceptors of the frog, Rana pipiens. J. Comp. Physiol. A 157: 657-665.

Wolbarsht, M. L., and V. G. Dethier (1958) Electrical activity in the chemoreceptors of the blowfly. J. Gen. Physiol. 42: 393-412. 